\title{
Autologous transfusion of "old" red blood cells-induced M2 macrophage polarization through IL-10-Nrf2-H0-1 signaling complexes
}

\author{
Zhen-Zhou Li Li, ,A-E, Zi-Wei Zhang 3,A-D, Huan Wang 2,B,C,E,F, Yong-Quan Chen 2,B,C,E, \\ Xiao-Fang Zhou ${ }^{2, B, C}$, Li-Shuang Duan ${ }^{2, C, E}$, Xiao-Xiao Wang ${ }^{2, E}$, Feng Xu ${ }^{4, E}$, Jian-Rong Gu0 ${ }^{1,2, B, F}$ \\ 1 Shanghai Pudong New Area Gongli Hospital Training Base, Ningxia Medical University, China \\ 2 Department of Anesthesiology, Gongli Hospital, Second Military Medical University, Shanghai, China \\ ${ }^{3}$ Department of Anesthesiology, Yijishan Hospital, Wannan Medical College, Wuhu, China \\ ${ }^{4}$ Department of Anesthesiology, Anhui Province No. 2 People's Hospital, Hefei, China \\ A - research concept and design; $\mathrm{B}$ - collection and/or assembly of data; $\mathrm{C}$ - data analysis and interpretation; \\ $D$ - writing the article; $E$ - critical revision of the article; $F$ - final approval of the article
}

\section{Address for correspondence}

Jian-Rong Guo

E-mail: gj8259@yeah.net

\section{Funding sources}

Key Disciplines Group Construction Project of Pudong Health Bureau of Shanghai (grant No. PWZxq 2017-10) and National Natural Science Foundation of China (grant No. 81870147).

\section{Conflict of interest}

None declared

Received on September 30, 2019

Reviewed on February 27, 2020

Accepted on May 1, 2020

Published online on August 5, 2020

Cite as

Li ZZ, Zhang ZW, Wang H, et al. Autologous transfusion of "old" red blood cells-induced M2 macrophage polarization through IL-10-Nrf2-H0-1 signaling complexes. Adv Clin Exp Med. 2020;29(7):833-840. doi:10.17219/acem/121924

DOI

10.17219/acem/121924

Copyright

Copyright by Author(s)

This is an article distributed under the terms of the

Creative Commons Attribution 3.0 Unported (CC BY 3.0)

(https://creativecommons.org/licenses/by/3.0/)

\begin{abstract}
Background. Red blood cell (RBC) transfusion is associated with systemic inflammation and immune suppression as adverse outcomes.
\end{abstract}

Objectives. To investigate the immunomodulatory function of the transfused autologous RBC in altering pro-inflammatory and immunosuppressive effects.

Material and methods. A total of 24 Sprague Dawley male rats were randomly divided into 3 groups ( $n=8$ in each group). Group 1 did not receive blood transfusions, while the other 2 groups of rats separately received transfusion of RBC stored for 14 days (group 2) and 35 days (group 3). The rats were treated with HO-1 inhibitor, $\mathrm{HO}$-1 inducer and nuclear factor erythroid 2-related factor 2 (Nrf2) activator after they separately received autologous transfusion of RBC that were cryopreserved for 14 days or 35 days. The blood samples of the rats were collected $12 \mathrm{~h}$ after the transfusion, and the macrophage phenotype of $\mathrm{M} 1$ and $\mathrm{M} 2$ were analyzed with flow cytometry (FCM). Also, the surface protein expression of CD68 and CD200R in macrophages were analyzed and the inflammatory signals in the serum were measured with enzyme-linked immunosorbent assay (ELISA). Moreover, the location and expression of proteins heme oxygenase 1 (H0-1), arginine 1 (Arg-1) and nitric oxide synthase 2 (NOS2) in macrophage were detected with immunofluorescence (IF).

Results. Autologous transfusion of long-time stored ("old") RBC promoted macrophage polarization to M2 phenotype and upregulated the expression of its surface proteins CD68 and CD200R. The pro-inflammatory cytokines tumor necrosis factor a (TNF- $a$ ), interleukin (IL)-6, IL-1 3 , and IL-18 were inhibited, and the secretion of NOS isoforms (iNOS) in serum was reduced with blood transfusion; contrarily, the production of IL-10 and CCL22 was increased. Additionally, H0-1, Arg-1 and NOS2 proteins were located in the cytoplasm, and H0-1 and Arg-1 proteins were highly expressed in macrophage, while the expression of protein NOS2 was low. Moreover, Nrf2, H0-1 and Arg-1 proteins were upregulated in macrophage after receiving "old" RBC transfusion.

Conclusions. Autologous transfusion of "old" RBC drove the macrophage phenotype toward M2 macrophages and induced immunosuppressive effects through the IL-10-NRF2-HO-1 signals.

Key words: immunosuppressive treatment, red blood cell transfusion, M2 macrophage, IL-10-NRF2-HO-1 signals 


\section{Introduction}

Blood transfusion is widely used in clinical practice; it is estimated that the transfusion of red blood cells (RBC) is the major type, accounting for nearly $50 \%$ of transfusions. ${ }^{1}$ The obtained RBC cryopreserved at $4 \pm 2{ }^{\circ} \mathrm{C}$ for 35-42 days were recognized as safe ${ }^{2}$; however, in recent years, more adverse effects have occurred after blood transfusions, ${ }^{3-5}$ which is why the storage lesions of $\mathrm{RBC}$ have attracted more attention. Even though low-temperature storage slows down the metabolism of RBC, studies have revealed that the structure and physiological and immunological characteristics of the long-time stored RBC (also named "old” RBC) have changed. The research of Karon et al. showed that the levels of RBC membrane 2,3-diphosphoglycerate (2,3-DPG) had decreased during storage. ${ }^{6}$ The proteomic analysis identified the membrane structural proteins in RBC, such as spectrin, band 3 and band 4.1, which were changed. ${ }^{7}$ Moreover, the stored RBC induced hemolysis and the release of free heme, hemoglobin and free iron, which play important roles in immunomodulation. ${ }^{8}$ The free heme was not only sequestered by plasma haptoglobin, but also initiated the Fenton reaction to induce the release of iron ${ }^{9}$; then, the accumulation of iron induced the generation of reactive oxygen species (ROS) and radical chains, which thus led to tissue damage and inflammation. ${ }^{10,11}$ Additionally, the free heme act as a pro-inflammatory factor engaged in the immune response of monocytes, macrophages, Tregs, and endothelial cells. ${ }^{12,13}$ A study showed that free heme mediated macrophage polarization and shaped the expression of M1 and M2 markers. ${ }^{14}$ More interestingly, the microvesicles were also found in the stored "old" RBC, which participated in the activity of endothelial activation, ${ }^{15}$ blood coagulation ${ }^{16}$ and immunomodulation. ${ }^{17}$ Hence, the variability of stored RBC is closely associated with systemic proinflammatory and immunosuppressive effects in blood post-transfusion.

The "old" RBC transfusion also burdened the phagocytic ability of monocytes and macrophages. A study conducted by Dinkla et al. identified that the storage-induced autoantibodies in RBC membrane accelerated phagocytosis by macrophages. ${ }^{18}$ This correspondingly resulted in heme accumulation in macrophages, which in turn shaped the macrophages toward M2 phenotype through upregulating the heme oxygenase 1 (HO-1) and promoted the secretion of interleukin (IL)-10. ${ }^{19}$ Therefore, the stored RBC transfusion induced adverse effects, owing to systemic inflammation and immune suppression. The aim of this study was to investigate the immunomodulation effects on macrophage of the "old" RBC transfusion in animal study and its related mechanism.

\section{Material and methods}

\section{Material}

A total of 24 Sprague Dawley male rats, 6-7 weeks old, $205.45 \pm 20.55$ g (Shanghai Lab Animal Research Center, Shanghai, China); animal blood-taking needles (G-5mm, Braintree Scientific, USA); uncoated vacutainer tube with sodium citrate (Greiner Bio-One International $\mathrm{GmbH}$, Kremsmünster, Austria); peripherally inserted central catheter (PICC; Bard Access Systems, Inc., Salt Lake City, USA); blood transfusion tube needle (Bard Access Systems, Inc.); plastic storage bag prefilled with mannitol adenine phosphate (MAP) storage solution (Sichuan Nigale Biomedical Co. Ltd., Chengdu, China); HO-1 inducer-cobalt protoporphyrin (CoPP, catalog No. ALX-430-076-M025; Jiangxi Haoran BioPharma Co., Ltd., Nanchang, China); HO-1 inhibitor-zinc protoporphyrin IX (ZnPP IX, catalog No. 282820-50MG; Jiangxi Haoran Bio-Pharma Co,. Ltd.); and Nrf2 activatoroltipraz (catalog No. MB2316; Dalian Meilun Biotech Co., Ltd., Dalian, China).

This study conformed to the standards of the animal ethics committee.

\section{Sample collection}

Following transfusion, the fresh whole bloods were collected from the aorta abdominalis after the 10 rats were anesthetized with $2 \%$ isoflurane and sacrificed. The obtained blood was leukocyte-depleted with automatic leukocyte filter (catalog No. PXL8Y; Shanghai Jiading Photoelectric Instrument Co., Ltd., Shanghai, China) The left $\mathrm{RBC}$ units were then all stored in a plastic storage bag and separately stored at $4^{\circ} \mathrm{C}$.

\section{Transfusion model}

The 24 Sprague Dawley male rats were maintained in adapted cultivation at $22-25^{\circ} \mathrm{C}$, in a $50-70 \%$ relative humidity environment with a 12-hour light/dark cycle for 2 weeks. Then, they were divided into 3 groups: group 1 did not receive blood transfusions, while the other 2 groups of rats separately received transfusion of $\mathrm{RBC}$ stored for 14 days (group 2) and 35 days (group 3 ). The collected RBC samples stored for 14 and 35 days were separately transported in a blood transfusion tube needle connected with a PICC, which was injected into the vein. After a six-hour blood transfusion, the rats were anesthetized with $2 \%$ isoflurane and sacrificed; then, the blood samples were collected from the eyeball with an uncoated vacutainer tube with sodium citrate.

\section{Flow cytometry}

The blood samples collected from the eyeball were diluted 1:3 with phosphate-buffered saline (PBS) containing heparin, and $3 \mathrm{~mL}$ of Ficoll-Hypaque solution (Tianjin 
Haoyang Commercial Co., Ltd., Tianjin, China) was slowly added into the tube, which was then centrifuged twice at 3,000 $\times \mathrm{g}$ for $15 \mathrm{~min}$ at $4^{\circ} \mathrm{C}$. Afterward, the supernatant in the middle class was transferred into a new tube, where its density was adjusted at $5 \times 10^{5}-3 \times 10^{6}$ with Dulbecco's modified Eagle's medium (DMEM) containing $10 \%$ fetal calf serum (FCS). Later, we firstly analyzed the percentage of macrophages. The steps were as follows: the monocytes were stained with allophycocyanin (APC)-conjugated monoclonal antibody against mouse CD16 (catalog No. 170168-41; eBioscience, Thermo Fisher Scientific, Waltham, USA); subsequently, the phycoerythrin (PE)-conjugated monoclonal antibody against mouse CD68 (catalog No. MA5-23572; Invitrogen, Carlsbad, USA) and CD200R (catalog No. 12-9201-42; eBioscience) were separately stained with the monocytes for $1 \mathrm{~h}$ at $4^{\circ} \mathrm{C}$. Afterward, the cells were washed with PBS and the second antibody - anti-mouse immunoglobulin G (IgG) - was added. Finally, the monocytes were fixed with paraformaldehyde for $30 \mathrm{~min}$ and analyzed with FACSCalibur ${ }^{\mathrm{TM}}$ (eBioscience). We obtained the monocyte medium and stained the primary conjugated antibody as above. The Fc was blocked and the isotypic antibodies were used as a control; similarly, the surface proteins CD68 and CD200R were analyzed with FACSCalibur.

\section{ELISA assay}

The blood samples collected from the eyeball were centrifuged twice at $3,000 \times \mathrm{g}$ for $15 \mathrm{~min}$ at $4^{\circ} \mathrm{C}$; then, the supernatants were transferred into a new tube and analyzed for the inflammatory-associated molecules of tumor necrosis factor $\alpha$ (TNF- $\alpha$; catalog No. 70-ab35884-050; MultiSciences Biotech Co., Ltd., Hangzhou, China), IL-1 $\beta$ (catalog No. 70-ab33591-050; MultiSciences Biotech Co., Ltd.), IL-6 (catalog No. 70-ab36529-050; MultiSciences Biotech Co., Ltd.), IL-10 (catalog No. 70-ab33577-050; MultiSciences Biotech Co., Ltd.), nitric oxide synthase isoform (iNOS; catalog No. 93-E4649-100; BioVision Inc., Milpitas, USA), IL-18 (catalog No. 70-ab33588-050; MultiSciences Biotech Co., Ltd.), and CCL22 (catalog No. 70-ab2243-050; MultiSciences Biotech Co., Ltd.) with enzyme-linked immunosorbent assay (ELISA) kits according to the manufacturer's instructions.

\section{Immunofluorescence}

The blood samples collected from the eyeball were diluted 1:3 with PBS containing heparin, and $3 \mathrm{~mL}$ of FicollHypaque solution (Tianjin Haoyang Commercial Co., Ltd.) was slowly added into the tube, which were then centrifuged twice at $3000 \times \mathrm{g}$ for $15 \mathrm{~min}$ at $4^{\circ} \mathrm{C}$. Afterward, the supernatants in the middle class were transferred into a new tube, and the monocytes were cultivated with DMEM containing $10 \%$ FCS and and cell slides were made in each group.
Afterwards, the cell slides were permeabilized with $0.1 \%$ TritonX-100 for $5 \mathrm{~min}$, and then they were blocked with $10 \%$ bovine serum albumin (BSA) for $1 \mathrm{~h}$ at room temperature. After that, the primary antibodies HO-1 (catalog No. ab13248; Abcam, Cambridge, UK), arginine 1 (Arg-1; cata$\log$ No. 43933; Cell Signaling Technology, USA) and nitric oxide synthase 2 (NOS2; catalog No. ab15323; Abcam) were separately immunoblotted in the cells for $12 \mathrm{~h}, 4^{\circ} \mathrm{C}$; then, the cells were incubated with anti-mouse IgG antibody for $1 \mathrm{~h}$ at $37^{\circ} \mathrm{C}$. Thereafter, the cell slides were washed with PBS 3 times, and then the cell nucleus were dyed with 4,6-diamidino-2-phenylindole (DAPI) and mounted on the cell slide with anti-fade mounting medium; finally, the stained images were examined under confocal microscopy (Leica Camera AG, Wetzlar, Germany).

\section{Western blot}

The 60 rats after adapted cultivation were divided into 3 groups: group $1(n=20)$ did not receive a blood transfusion, while the rats in the other 2 groups (group 2: $\mathrm{n}=20$ and group 3: $\mathrm{n}=20$ ) separately received a transfusion of RBC stored for 14 days (group 2) and 35 days (group 3). Meanwhile, the rats from each of the 3 parts were divided into 4 subgroups ( $\mathrm{n}=5$ in each subgroup) - control subgroup, HO-1 inhibitor administration (ZnPP IX) subgroup, $\mathrm{HO}-1$ inducer administration (CoPP) subgroup, and Nrf2 activator administration (oltipraz) subgroup. After $12 \mathrm{~h}$, the rats were anesthetized with $2 \%$ isoflurane and sacrificed. The blood samples were collected from each rat from the aorta abdominalis and then diluted 1:1 with PBS; subsequently, they were centrifuged and the monocytes were isolated with Ficoll-Hypaque solution. Thereafter, the total proteins of the monocytes were extracted with radioimmunoprecipitation assay (RIPA) buffer containing phenylmethylsulfonyl fluoride (PMSF), and then they were denatured and the protein concentration was evaluated. Afterward, the proteins were isolated with sodium dodecyl sulfate (SDS) polyacrylamide gels and the gels were transferred to polyvinylidene difluoride (PVDF) membranes. They were then blocked with $5 \%$ fat-free milk for $1 \mathrm{~h}$; subsequently, the protein membranes were separately incubated with primary antibodies: anti-HO-1 (catalog No. ab13248; Abcam), anti-Arg-1 (catalog No. 43933, Cell Signaling Technology, Danvers, USA) and anti-Nrf2 (catalog No. ab89443; Abcam) for $12 \mathrm{~h}$ at $4^{\circ} \mathrm{C}$. Afterward, the protein membranes were incubated with anti-mouse IgG antibody for $1 \mathrm{~h}$. The glyceraldehyde-3-phosphate dehydrogenase (GAPDH) protein was used as control. Finally, the electrochemiluminescence (ECL) detection reagents were added into the protein bands, which were visualized with a bandscan instrument (Cytiva, Marlborough, USA), while the gray intensity of the proteins was analyzed with ImageJ software (National Institutes of Health, Bethesda, USA). 

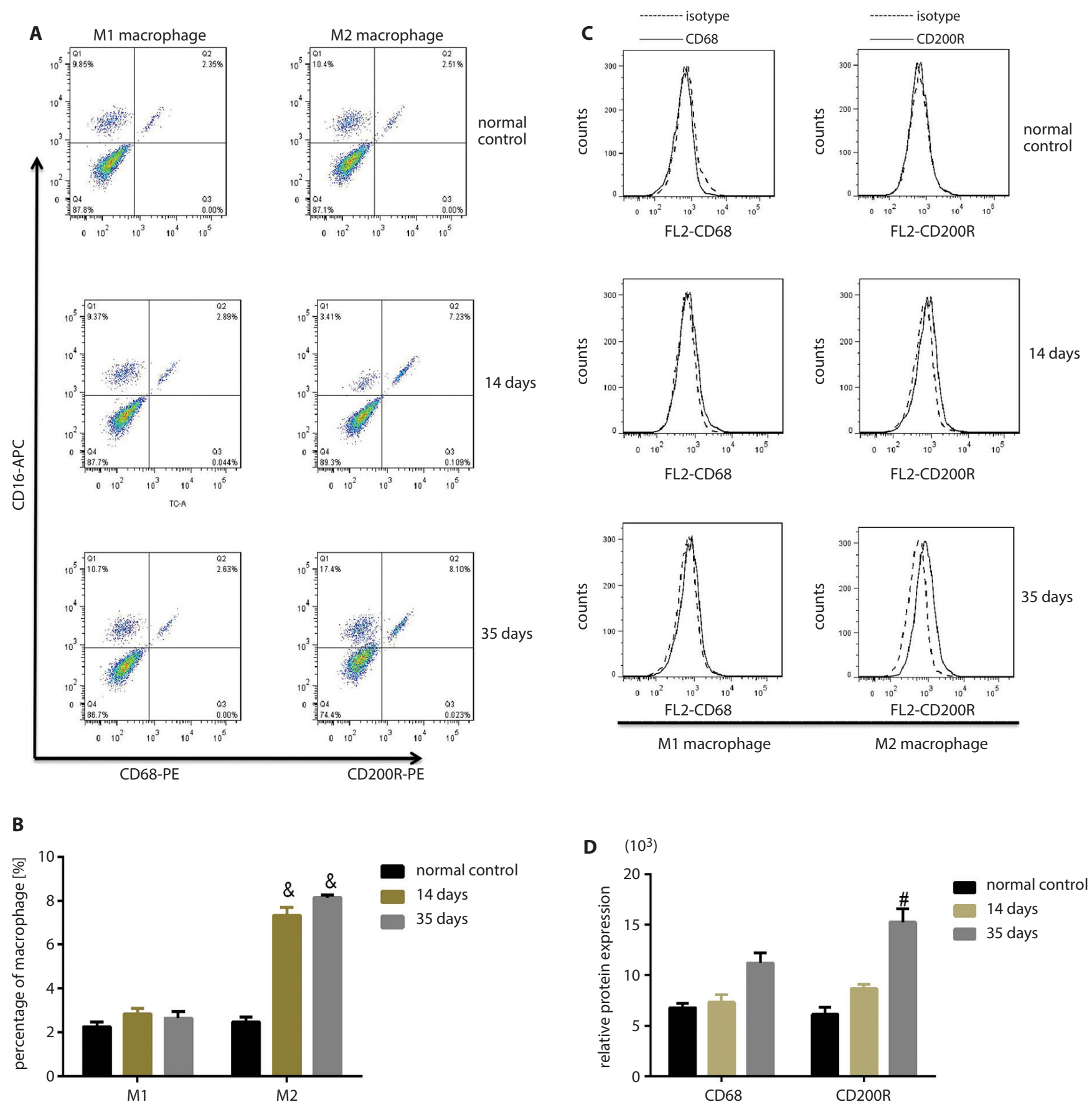

Fig. 1. Flow cytometry (FLC) sorted the macrophage phenotype (A) and detected the surface proteins expression of CD68 and CD200R (C). Blood transfusion shifted the macrophage to M2 phenotype $-{ }^{\&} p<0.05$ of M2 percentage with blood transfusion vs without blood transfusion (B). The surface protein CD200R was significantly upregulated - ${ }^{*} \mathrm{p}<0.05$ with a transfusion of blood stored 35 days vs with a transfusion of blood stored 14 days and without a blood transfusion (D)

\section{Statistical analysis}

The data was analyzed with the IBM SPSS Statistics for Windows software, v. 19.0 (IBM Corp., Armonk, USA), and the one-way analysis of variance (ANOVA) method was applied for analyzing the group difference. A p-value of $<0.05$ was considered the statistically significant difference. The graphs were depicted with GraphPad Prism v. 6.0 software (GraphPad Software Inc., San Diego, USA), and presented as means \pm standard error of means ( $\mathrm{M} \pm \mathrm{SEM})$. 


\section{Results}

\section{Autologous transfusion of "old" RBC induced M2 macrophage polarization}

After the rats received transfusion of RBC stored for 14 days or 35 days, the flow cytometry (FCM) results showed that the percentage of M2 phenotype macrophage was obviously increased after receiving a blood transfusion (Fig. 1A,B). The surface proteins CD68 in M1 phenotype macrophage and CD200R in M2 phenotype macrophage were also varied. Results revealed that the protein CD200R was highly expressed on M2 macrophage, while the protein CD68 was also upregulated, but the extent was not as evident as in the case of CD200R (Fig. 1C,D); hence, as a whole, the $\mathrm{RBC}$ transfusion shifted the macrophage toward to M2 phenotype.

\section{Autologous transfusion promotes} the secretion of pro-inflammatory factors, including IL-6, IL-1 $\beta$, TNF- $\alpha$, iNOS, IL-10,

\section{IL-18, and CCL22}

The pro-inflammatory cytokines TNF- $\alpha$, IL-6, IL-1 $\beta$, and IL-18 were decreased with transfusion of blood stored for 35 days, while its amount was increased with a transfusion of RBC stored for 14 days. The variation tendency of iNOS was similar to the pro-inflammatory cytokines, which was consistent with the activity of iNOS involved in inflammation and enhancing the synthesis of proinflammatory mediators (Fig. 2). Conversely, the secretion of anti-inflammatory cytokine IL-10 and chemokine CCL22 was increased in blood after transfusion (Fig. 2). The exent of anti-inflammatory cytokine IL-10

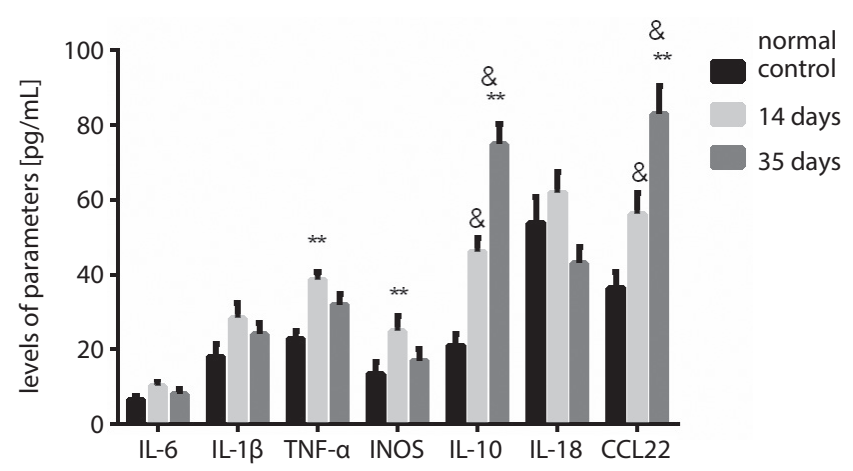

Fig. 2. The ELISA assay detected the amount of inflammatory molecules - ${ }^{* *} \mathrm{p}<0.05$ of TNF-a, iNOS, IL-10, and CCL22 with a transfusion of blood stored 14 days vs without a blood transfusion; ${ }^{p} \mathrm{p}<0.05$ of IL-10 and CCL22 with a transfusion of blood stored 35 days vs with a transfusion of blood stored 14 days

and chemokine CCL22 secretion was more obvious with a transfusion of RBC stored for 35 days. These results demonstrated that autologous transfusion of blood could increase the inflammatory response.

\section{Autologous transfusion directs the subcellular distribution of HO-1, Arg-1 and NOS2 to the cytoplasm}

A few studies reported that $\mathrm{HO}-1$ could mediate the antiinflammatory phenotype of macrophage through regulating NRF $2 .{ }^{20}$ In this study, the proteins HO-1, Arg- 1 and NOS2 were found to be located in the cytoplasm of macrophage; HO-1 and Arg-1 had high expression (Fig. 3). Therefore, we proposed that autologous transfusion of blood exerted its function through the redistribution of $\mathrm{HO}-1$, Arg-1 and NOS2.
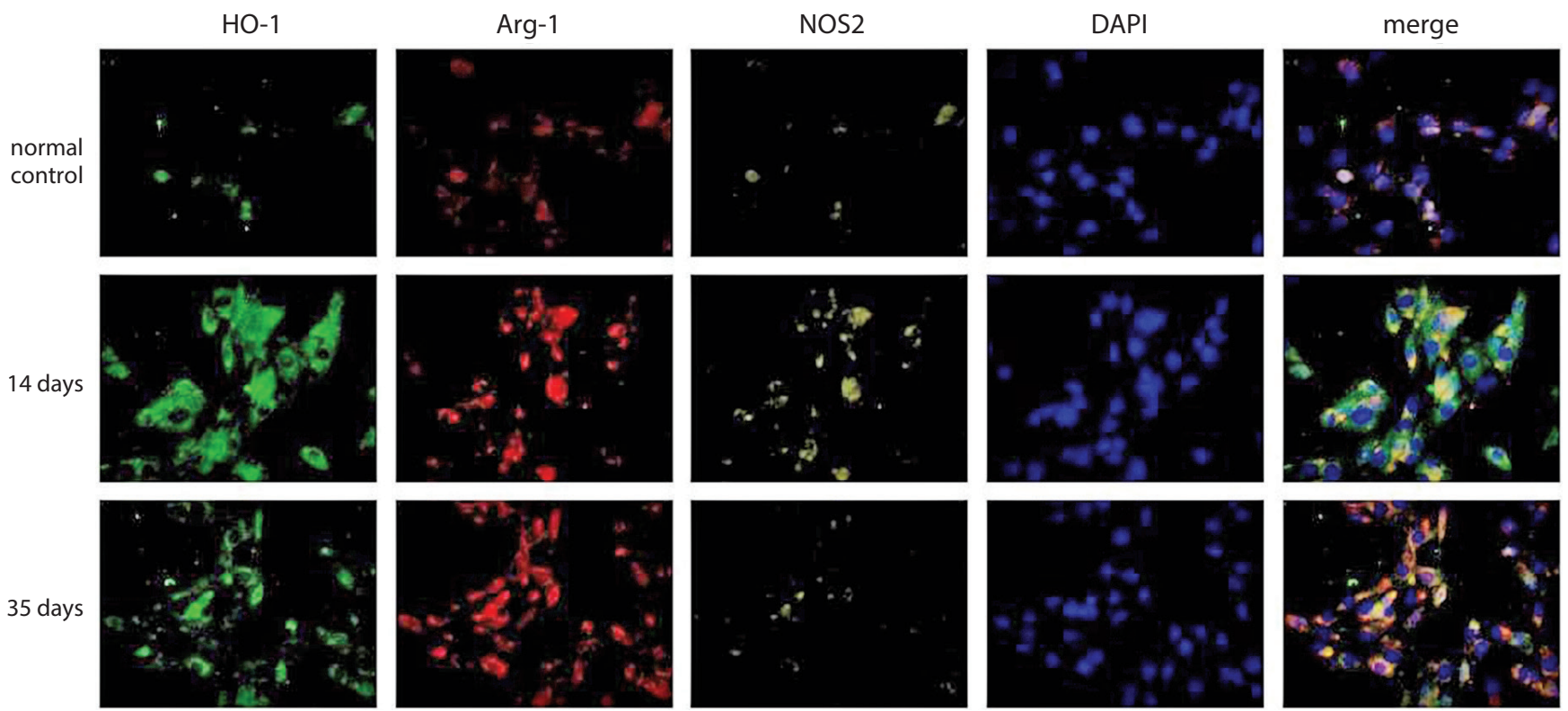

Fig. 3. Immunofluorescence (IF) identified the proteins HO-1, Arg-1 and NOS2. The proteins HO-1, Arg-1 and NOS2 located in the cytoplasm of macrophage and proteins HO-1 and Arg-1 with a high expression, while protein NOS2 did not have a high level of expression 
A

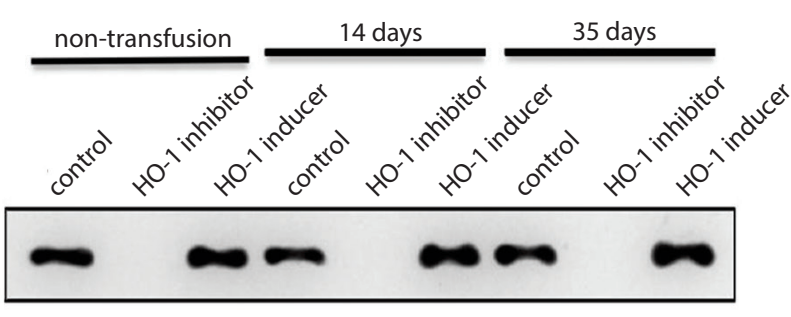

Arg-1

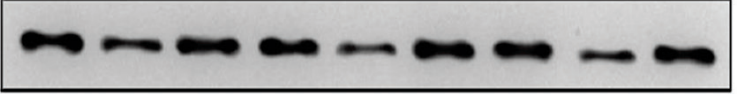

GAPDH
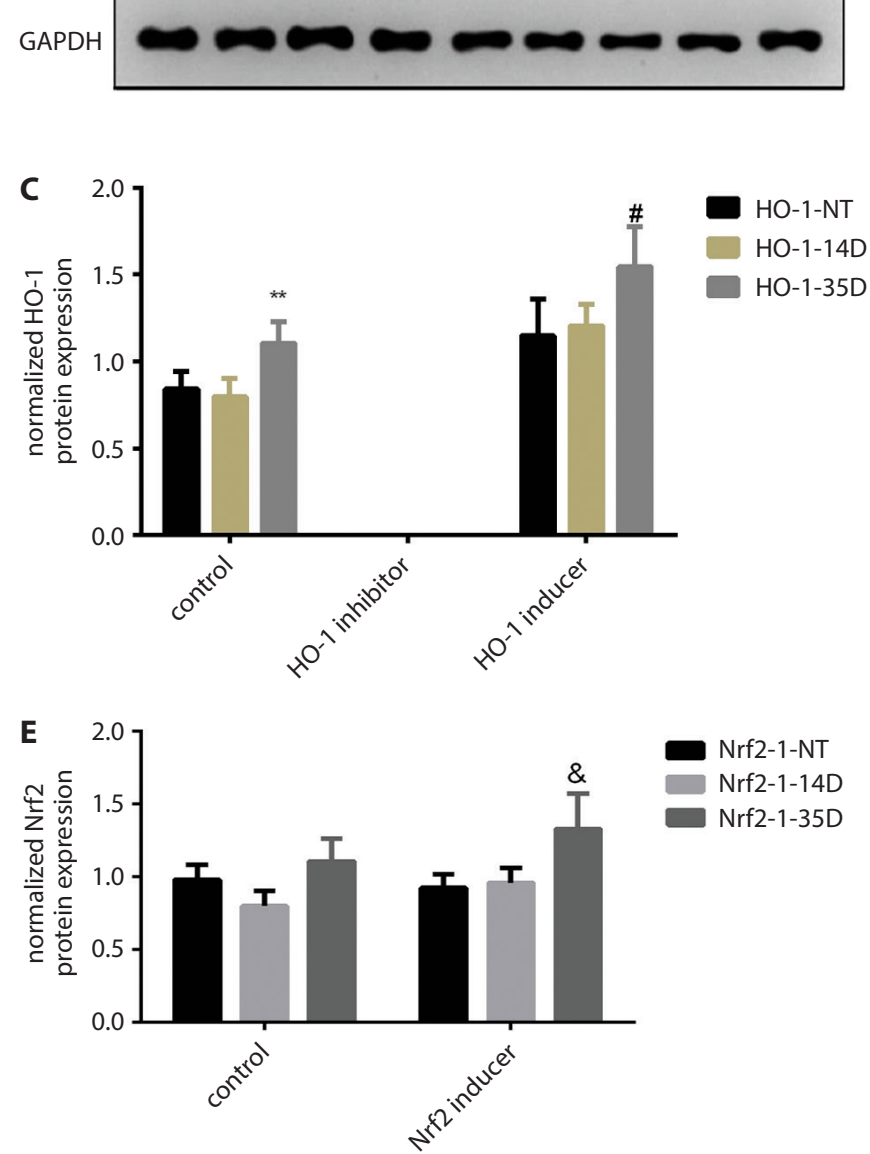

B

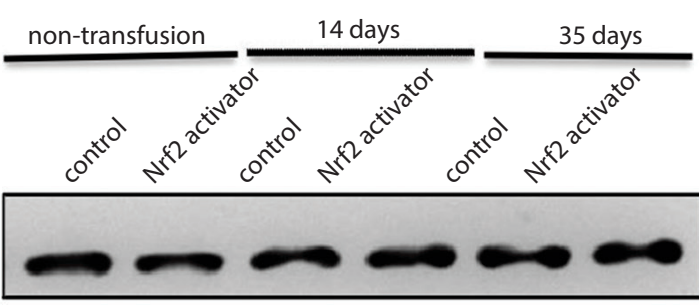

HO-1

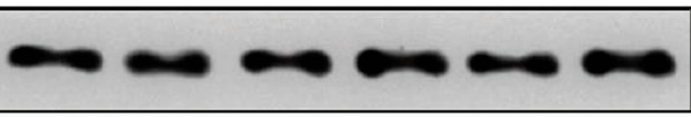

GAPDH

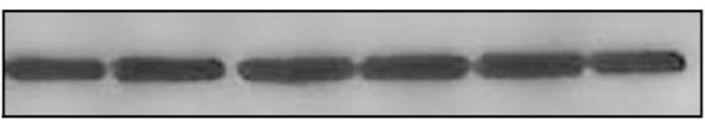

D
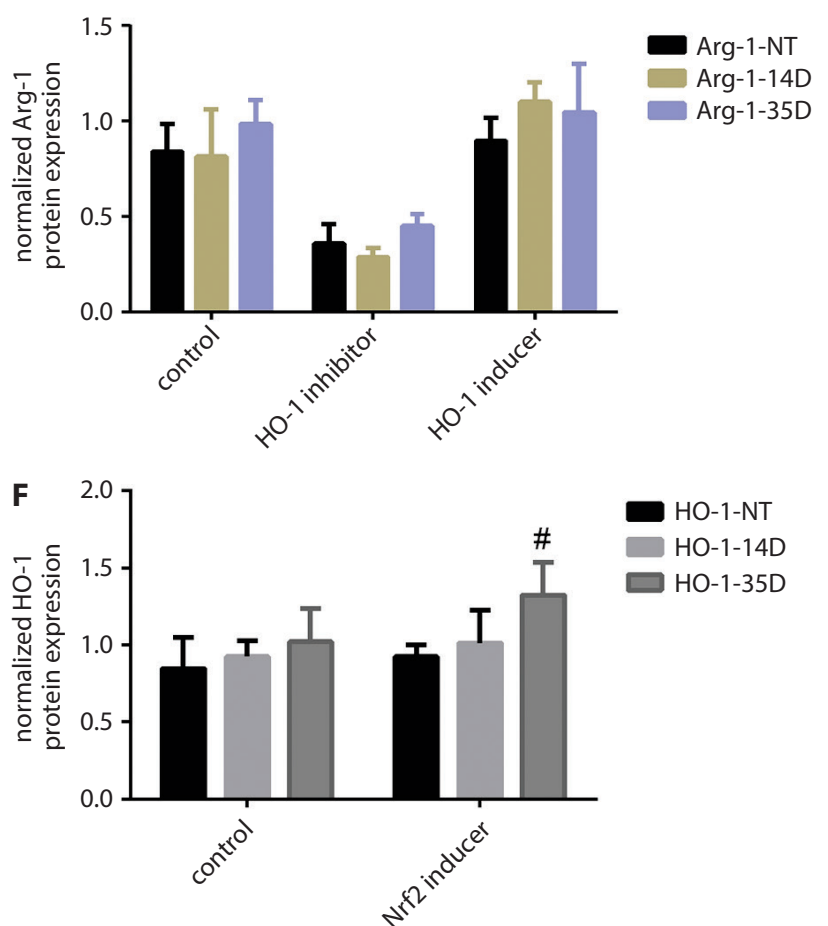

Fig. 4. A and B. The expression of proteins Nrf2, $\mathrm{HO}-1$ and Arg-1 in macrophages of rats with or without blood transfusion analyzed with western blot. The HO-1 inhibitor (ZnPP IX), HO-1 inducer (CoPP) and Nrf2 activator (oltipraz) were all administrated in rats with or without blood transfusion. C. The upregulated $\mathrm{HO}-1$ with a transfusion of blood stored 35 days $-{ }^{* *} \mathrm{p}<0.05$ vs with a transfusion of blood stored 14 days; ${ }^{*} \mathrm{p}<0.05$ vs without a blood transfusion. D. With HO-1 inhibitor (ZnPP IX) administration, the protein Arg-1 downregulated. E and F. The upregulated Nrf2, HO-1 with a transfusion

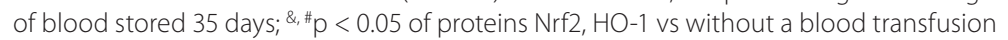

\section{HO-1 inhibitor significantly repressed the protein expression of $\mathrm{HO}-1$ while promoting the expression of Arg-1, but NRF2 activator has no effects on NRF2 and HO-1 after autologous transfusion}

With the HO-1 inhibitor administration (ZnPP IX), HO-1 inducer administration (CoPP) and Nrf2 activator administration (oltipraz), we found that the expression of proteins Nrf2, HO-1 and Arg-1 was significantly changed with a transfusion of RBC stored for 35 days, compared with the rats who did not receive blood transfusion or received a transfusion of RBC stored for 14 days (Fig. 4A,B). The inhibition of HO-1 affected the expression of protein Arg-1 (Fig. 4C,D), and to some extent, the activation of HO-1 and Nrf2 promoted the expression of proteins Nrf2, HO-1 and Arg-1 with a transfusion of RBC stored for 35 days (Fig. 4E,F). Hence, an autologous transfusion of "old" RBC induced immunosuppressive effects through 
the IL-10-Nrf2-HO-1 signals. These results indicated that an autologous transfusion of blood functioned through HO-1 and Arg-1, but not through Nrf2.

\section{Discussion}

The mechanism responsible for the adverse outcomes of systemic inflammation and immune suppression was controversial. In our study, we found that the transfusion of "old" $\mathrm{RBC}$ in rats induced the polarization of macrophages to M2 phenotype and upregulated their surface marker CD200R expression. The levels of anti-inflammatory signals IL-10 and CCL22 were also enhanced. More interestingly, the pro-inflammatory signals TNF- $\alpha$, iNOS, IL-6, IL-1 $\beta$, and IL-18 were increased after a transfusion of RBC stored for 14 days, while their levels were decreased after a transfusion of RBC stored for 35 days. This feature might be caused by the regulation of both pro-inflammation and anti-inflammation response. However, a RBC transfusion in a human study showed that there was no increase in pro-inflammatory cytokines, except for a small increase in non-transferrin-bound iron (NTBI). ${ }^{21}$ However, a rat model with of blood transfusion suggested that a pro-inflammatory cytokine storm and an increase of NTBI occurred. ${ }^{22}$ Hence, the debatable results may be caused by species difference and the limitation of study samples. The transfusion of stored blood resulting in increased hemolysis and erythrophagocytosis features were increasingly confirmed, which lead to a significant increase in serum iron, free heme and bilirubin levels. ${ }^{23-25}$ The free heme was suggested as a pro-inflammatory signal, involved in the activity of IL-1 and TNF-a, and ROS generation. ${ }^{26,27}$ Hence, this may be the reason that the pro-inflammatory signals TNF- $\alpha$, iNOS, IL-6, IL-1 $\beta$, and IL-18 were increased with the transfusion blood stored for 14 days. Moreover, HO-1 was suggested as a free heme scavenger, which had anti-inflammatory effects with a reduction of TNF-a, IL-8 and macrophage inflammatory protein (MIP)- $1 \beta$ levels and upregulation of IL-12. ${ }^{28}$ This evidence was in line with our study that the pro-inflammatory signals were decreased with a transfusion of blood stored for 35 days. On the other hand, studies also showed that $\mathrm{HO}-1$ was involved in the activity of polarizing M2 phenotype ${ }^{29,30}$; hence, this may be potentially involved in polarizing M2 phenotype.

With further studies, we found that HO-1, Arg-1, NOS2, and Nrf2 proteins were indeed engaged in M2 polarization. The HO-1, Arg-1 and Nrf2 proteins were obviously expressed in macrophages. This finding was consistent with the conclusion of Park et al. ${ }^{31}$ that $\mathrm{Nrf} 2$ was an essential regulator of HO-1 participating in antioxidative stressinduced inflammatory activity. Moreover, the results of M1 subtype marker molecule - NOS2 and M2 marker - Arg- $1^{32}$ further confirmed our finding that "old" RBC transfusion induced M2 phenotype polarization. Hence, the immunomodulation mechanism of our study was closely associated with Nrf2-HO-1 signals. Additionally, in our study, the secretion of CCL22 was also increased after transfusion. The CCL22 played roles in the trafficking of activated/effector T-lymphocytes. The study also showed that heme could drive the expansion of Tregs by inducing HO-1 expression in non-classical monocytes, ${ }^{33}$ so there is a sign that Tregs lymphocytes may also be involved in the immunosuppressive activity.

On the other hand, numerous studies have identified the cluster of differentiation (CD) molecules that are closely associated with the phagocytosis ability. The CD47, an integrin-associated protein of $\mathrm{RBC}$, was proved to be a selfmarker in deciding its phagocytosis by binding to the signal regulatory protein $\alpha(\operatorname{SIRP} \alpha)$ of the macrophage. ${ }^{34,35}$ Furthermore, the microvesicles of CD47, PS, CD55, and CD59 also shaped the phagocytosis of RBC. ${ }^{36,37}$ Therefore, we should further investigate the changes in $\mathrm{RBC}$, such as the change in membrane proteins, the secretion of $\mathrm{mi}$ crovesicles or spontaneous hemolysis, so as to more clearly reveal the mechanism of "old" RBC transfusion. Our study showed that the transfusion of "old" $\mathrm{RBC}$ in rats induced the polarization of macrophages to M2 phenotype through the IL-10-NFR2-HO-1 signals.

\section{ORCID iDs}

Zhen-Zhou Li (1) https://orcid.org/0000-0003-3774-7565 Zi-Wei Zhang (D) https://orcid.org/0000-0002-2140-4886 Huan Wang (D) https://orcid.org/0000-0003-3646-2656 Yong-Quan Chen (D) https://orcid.org/0000-0002-3525-1518 Xiao-Fang Zhou (D) https://orcid.org/0000-0002-9300-4478 Li-Shuang Duan (D) https://orcid.org/0000-0001-8510-7277 Xiao-Xiao Wang (D) https://orcid.org/0000-0003-4764-2281 Feng Xu (D) https://orcid.org/0000-0002-7750-7809 Jian-Rong Guo (D) https://orcid.org/0000-0002-3995-2995

\section{References}

1. Carson JL, Guyatt G, Heddle NM, et al. Clinical practice guidelines from the AABB: Red blood cell transfusion thresholds and storage. JAMA. 2016;316(19):2025-2035.

2. Dagur PK, McCoy JP. Collection, storage, and preparation of human blood cells. Curr Protoc Cytom. 2015;73:5.1.1-5.1.16. doi:10.1002/ 0471142956.cy0501s73

3. Muszynski JA, Spinella PC, Cholette JM, et al; Pediatric Critical Care Blood Research Network (Blood Net). Transfusion-related immunomodulation: Review of the literature and implications for pediatric critical illness. Transfusion. 2017;57(1):195-206.

4. Hod EA, Godbey EA. The outsider adverse event in transfusion: Inflammation. Presse Med. 2016;45(7-8 Pt 2):e325-e329.

5. van de Watering L. Red cell storage and prognosis. Vox Sang. 2011; 100(1):36-45.

6. Karon BS, Hoyer JD, Stubbs JR, Thomas DD. Changes in Band 3 oligomeric state precede cell membrane phospholipid loss during blood bank storage of red blood cells. Transfusion. 2009;49(7):1435-1442.

7. D'Amici GM, Rinalducci S, Zolla L. Proteomic analysis of RBC membrane protein degradation during blood storage. J Proteome Res. 2007;6(8):3242-3255.

8. Ozment CP, Mamo LB, Campbell ML, Lokhnygina Y, Ghio AJ, Turi JL. Transfusion-related biologic effects and free hemoglobin, heme, and iron. Transfusion. 2013;53(4):732-740.

9. Banerjee S. Glyoxal-induced modification enhances stability of hemoglobin and lowers iron-mediated oxidation reactions of the heme protein: An in vitro study. Int J Biol Macromol. 2018;107(Pt A): 494-501.

10. Spitalnik SL. Stored red blood cell transfusions: Iron, inflammation, immunity, and infection. Transfusion. 2014;54(10):2365-2371. 
11. Baek JH, Yalamanoglu A, Gao Y, et al. Iron accelerates hemoglobin oxidation increasing mortality in vascular diseased guinea pigs following transfusion of stored blood. JCI Insight. 2017;2(9):e93577.

12. Dutra FF, Bozza MT. Heme on innate immunity and inflammation. Front Pharmacol. 2014;5:115.

13. Haldar M, Kohyama M, Yick-Lun So A, et al. Heme-mediated SPI-C induction promotes monocyte differentiation into iron-recycling macrophages. Cell. 2014;156(6):1223-1234.

14. Vinchi F, Costa da Silva M, Ingoglia G, et al. Hemopexin therapy reverts heme-induced pro-inflammatory phenotypic switching of macrophages in a mouse model of sickle cell disease. Blood. 2016;127(4): 473-486.

15. Straat M, van Hezel ME, Böing A, et al. Monocyte-mediated activation of endothelial cells occurs only after binding to extracellular vesicles from red blood cell products, a process mediated by $\beta$-integrin. Transfusion. 2016;56(12):3012-3020.

16. Rubin O, Delobel J, Prudent M, et al. Red blood cell-derived microparticles isolated from blood units initiate and propagate thrombin generation. Transfusion. 2013;53(8):1744-1754.

17. Straat M, Böing AN, Tuip-De Boer A, Nieuwland R, Juffermans NP. Extracellular vesicles from red blood cell products induce a strong pro-inflammatory host response, dependent on both numbers and storage duration. Transfus Med Hemother. 2016;43(4):302-305.

18. Dinkla S, Novotný VMJ, Joosten I, Bosman GJCGM. Storage-induced changes in erythrocyte membrane proteins promote recognition by autoantibodies. PLoS One. 2012;7(8):e42250.

19. Yazdanbakhsh K, Bao W, Zhong H. Immunoregulatory effects of stored red blood cells. Hematology Am Soc Hematol Educ Program. 2011;2011:466-469.

20. Hod EA, Brittenham GM, Billote GB, et al. Transfusion of human volunteers with older, stored red blood cells produces extravascular hemolysis and circulating non-transferrin-bound iron. Blood. 2011; 118(25):6675-6682.

21. Hod EA, Zhang N, Sokol SA, et al. Transfusion of red blood cells after prolonged storage produces harmful effects that are mediated by iron and inflammation. Blood. 2010;115(21):4284-4292.

22. Hult A, Malm C, Oldenborg PA. Transfusion of cryopreserved human red blood cells into healthy humans is associated with rapid extravascular hemolysis without a pro-inflammatory cytokine response. Transfusion. 2013;53(1):28-33.
23. Knutson MD, Vafa MR, Haile DJ, Wessling-Resnick M. Iron loading and erythrophagocytosis increase ferroportin 1 (FPN1) expression in J774 macrophages. Blood. 2003;102(12):4191-4197.

24. Youssef LA, Rebbaa A, Pampou S, et al. Increased erythrophagocytosis induces ferroptosis in red pulp macrophages in a mouse model of transfusion. Blood. 2018;131(23):2581-2593.

25. Hao K, Hanawa $H$, Ding $L$, et al. Free heme is a danger signal inducing expression of pro-inflammatory proteins in cultured cells derived from normal rat hearts. Mol Immunol. 2011;48(9-10):1191-1202.

26. Larsen R, Gouveia Z, Soares MP, Gozzelino R. Heme cytotoxicity and the pathogenesis of immune-mediated inflammatory diseases. Front Pharmacol. 2012;3:77.

27. Cherry AD, Piantadosi CA. Regulation of mitochondrial biogenesis and its intersection with inflammatory responses. Antioxid Redox Signal. 2015;22(12):965-976.

28. Naito Y, Takagi T, Higashimura Y. Heme oxygenase-1 and anti-inflammatory M2 macrophages. Arch Biochem Biophys. 2014;564:83-88.

29. Weis N, Weigert A, von Knethen A, Brüne B, Luo K. Heme oxygenase-1 contributes to an alternative macrophage activation profile induced by apoptotic cell supernatants. Mol Biol Cell. 2009;20(5):1280-1288.

30. ParkEJ, Kim YM, Park SW, et al. Induction of HO-1 through p38 MAPK/ Nrf2 signaling pathway by ethanol extract of Inula helenium L. reduces inflammation in LPS-activated RAW 264.7 cells and CLP-induced septic mice. Food Chem Toxicol. 2013;55:386-395.

31. Lu S, Li D, Xi L, Calderone R. Interplay of interferon-gamma and macrophage polarization during Talaromyces marneffei infection. Microb Pathogen. 2019;134:103594.

32. Zhong $\mathrm{H}$, Yazdanbakhsh K. Hemolysis and immune regulation. Curr Opin Hematol. 2018;25(3):177-182.

33. Hagmann M. A new way to keep immune cells in check. Science. 2000;288(5473):1945-1946.

34. Oldenborg PA. Role of CD47 as a marker of self on red blood cells. Science. 2000;288(5473):2051-2054.

35. Stachurska A, Dorman M, Korsak J, et al. Selected CD molecules and the phagocytosis of microvesicles released from erythrocytes ex vivo. Vox Sang. 2019;114(6):576-587.

36. van Manen $L$, Peters AL, van der Sluijs PM, Nieuwland $R$, van Bruggen R, Juffermans NP. Clearance and phenotype of extracellular vesicles after red blood cell transfusion in a human endotoxemia model. Transfus Apher Sci. 2019;58(4):508-511. 